UDC: 123.4

\title{
Simulation of Buffered Advection Diffusion of Calcium in a Hepatocyte Cell
}

\author{
Jagtap Y.D.*, Adlakha N. ${ }^{\dagger}$ \\ AMHD, S. V. National Institute of Technology, Ichchhanath \\ Surat, Gujarat 395007, India
}

\begin{abstract}
The calcium signaling is the basic and vital component of cell communication in almost all types of human and animal cells. All the vital functions of parenchymal cell of liver known as hepatocyte cell are regulated by this calcium signaling. The calcium concentration at specific levels are responsible for each of the various functions of the cell. The deeper understanding of the mechanisms and the factors affecting the calcium dynamics in a hepatocyte cell is vital for various clinical applications related to diseases of the liver. In this paper, mathematical model is proposed to study intracellular calcium dynamics in hepatocyte cell by incorporating the processes like diffusion, advection, buffering etc. The reaction advection diffusion equation has been employed for a two dimensional unsteady state case, to form an initial and boundary value problem. The initial and boundary conditions are formulated based on the physical conditions of cell. Finite volume method and Crank Nicolson scheme have been employed along spatial and temporal dimension respectively to obtain numerical solution. The impact of endogenous and exogenous buffers, advection and diffusion on calcium dynamics in hepatocyte cell has been studied with the help of numerical results. The rise and fall in spatio-temporal calcium concentration in hepatocyte cell in response to specific conditions of advection, diffusion and buffer concentrations is observed. These variations in spatio-temporal calcium concentrations are regulated in narrow range due to fine coordination among these processes of cell under normal environmental and physiological conditions. The proposed model gives better understanding of interrelationship and interdependence of these physical processes for fine coordination among them to maintain structure and functions of cell.
\end{abstract}

Key words: hepatocyte cell, advection, diffusion, calcium, buffer, finite volume method.

\section{INTRODUCTION}

All vital functions of hepatocyte cell like glycogenolysis, production of proteins, contraction of bile canaliculi, apoptosis, cell cycle, the movement of lysosomes and other vesicles in cytoplasm are regulated by ionized calcium $[1, \underline{2}]$. The hepatocyte cell is cubical in shape and its internal calcium stores like endoplasmic reticulum are more denser in apical region of cell. The $\mathrm{Ca}^{2+}$ ions released from gate of calcium channel in cytoplasm undergoes different physical mechanisms like transportation and buffering etc. The calcium transport takes place by the combination of advection and diffusion in cytoplasm. The advection is the transport of $\mathrm{Ca}^{2+}$ ions by bulk motion due to pressure, viscosity, gravity etc. It causes the movement of calcium

\footnotetext{
*yogitajagtap7886@gmail.com

†neeru.adlakha21@gmail.com
} 
ions in cross flow direction. While the transport of $\mathrm{Ca}^{2+}$ ions due to concentration gradient is known as diffusion. Besides this transportion activity, calcium concentration is also controlled by buffering mechanism. About $99 \%$ of calcium in cytoplasm binds with buffers to change their enzymatic properties $[\underline{3}, \underline{4}]$.

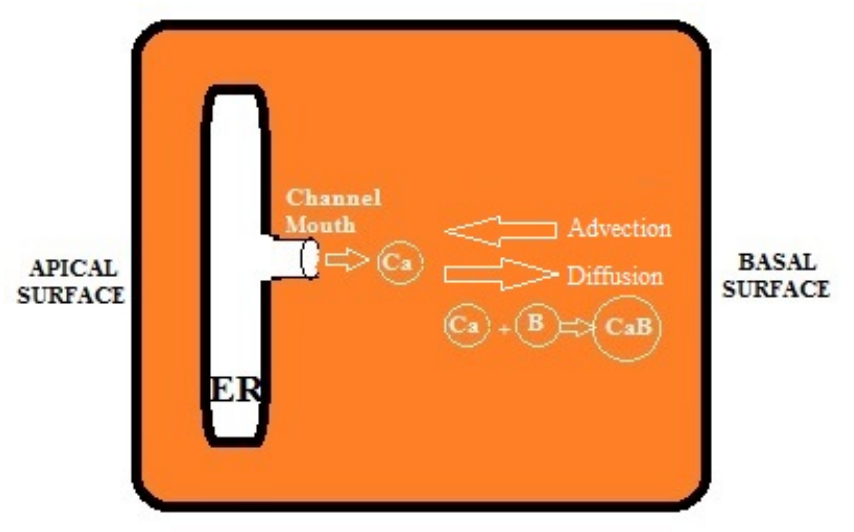

Fig. 1. Transport mechanisms in a hepatocyte cell.

It is evident from experimental investigation that calcium release always originates from specific region of the cell $[1, \underline{4}]$. The essential role of diffusion mechanism and buffers on the calcium dynamics is studied in cell with the help of mathematical model by M. S. Jafri [3]. The significance of numerical models to study biological processes involving diffusion and buffering is also discussed by past researchers [5]. The numerical study of calcium dynamics in the presence of excess buffer is reported in neuron cell $[\underline{6}, \underline{7}]$, fibroblast $[\underline{8}, \underline{9}]$, astrocyte $[10,11]$, oocyte [12], myocyte [13, 14] and hepatocyte cell [15] for one and two dimensional cases. The effect of calcium fluxes on spatio temporal variations of calcium concentration has been studied in neuron cell [16] and oocyte cell [17, 18]. Some attempts have been made to study the mechanics of calcium oscillations in pancreatic acinar cell $[\underline{19}, \underline{20}]$. Very few attempts are reported for the study of one dimensional advection diffusion of calcium in astrocyte [21], myocyte [22] and oocyte [23]. No any attempt have been observed to study of buffered advection diffusion of calcium in hepatocyte cell. In this paper a mathematical model is derived to study combined effect of advection diffusion of calcium in the presence of different buffers in two dimensions. The results are obtained to study organization of calcium concentration with time, in presence of different buffers with different buffer concentrations, at different cross flow velocities and various values of diffusion coefficient of calcium in cytoplasm. In the primary part mathematical model with auxiliary conditions is derived to incorporate all mechanisms under consideration. Then finite volume method is implemented and simulated in MATLAB at different values of biophysical parameters. Obtained results are discussed in later part of paper.

\section{MATHEMATICAL MODEL}

Advection diffusion of calcium in a hepatocyte cell is governed by the principle of superposition. Total calcium flux in the cell is sum of diffusive and advective flux. The process of advection diffusion in presence of excess buffer is described by following expression [19, 20],

$$
\begin{aligned}
& \text { Rate of change of calcium concentration in hepatocyte cell } \\
& =\text { Diffusive flux }+ \text { Advective flux }+ \text { Buffer effect. }
\end{aligned}
$$

The partial differential equation involving diffusion, advection and buffering mechanism is given by $[\underline{3}, \underline{24}]$, 


$$
\frac{\partial C}{\partial t}=D_{C}\left(\frac{\partial^{2} C}{\partial x^{2}}+\frac{\partial^{2} C}{\partial y^{2}}\right)-u \frac{\partial C}{\partial x}-k_{j}^{+}\left[B_{j}\right]_{\infty}\left(C-C_{\infty}\right)
$$

for

$$
0 \leq x \leq 20, \quad 0 \leq y \leq 20, \quad t \geq 0
$$

where $C$ is cytosolic calcium concentration. $[B]_{\infty}$ excess buffer concentration given by $\frac{K[B]_{T}}{K+C_{\infty}}$, where $K=\frac{k_{j}^{+}}{k_{j}^{-}}$is dissociation constant. The numerical values of biophysical parameters used for simulation are mentioned in Table $1[\underline{3}, \underline{24}]$.

Table 1. Numerical values of biophysical parameters

\begin{tabular}{|c|c|c|}
\hline Parameter & Description of parameter & Numerical value \\
\hline$D_{C}$ & Diffusion coefficient & $100-200 \mu \mathrm{m}^{2} / \mathrm{S}$ \\
\hline$u$ & Advection velocity & $10-20 \mu \mathrm{m} / \mathrm{s}$ \\
\hline$C_{\infty}$ & Stable calcium concentration & $0.1 \mu M$ \\
\hline$k_{j}^{+}$for EGTA & Buffer association constant & $1.5 \mu M^{-1} S^{-1}$ \\
\hline$K$ for EGTA & Dissociation constant & $0.2 \mu M$ \\
\hline$k_{j}^{+}$for Endogenous buffer & Buffer association constant & $50 \mu M^{-1} S^{-1}$ \\
\hline$K$ for Endogenous buffer & Dissociation constant & $10 \mu M$ \\
\hline$k_{j}^{+}$for BAPTA & Buffer association constant & $600 \mu M^{-1} S^{-1}$ \\
\hline$K$ for BAPTA & Dissociation constant & $0.17 \mu M$ \\
\hline$[B]_{T}$ & Total buffer concentration & $50-150 u p \mu M$ \\
\hline
\end{tabular}

\section{Auxiliary Conditions}

The shape of hepatocyte cell is cubical, having sides of length approximately $20 \mu \mathrm{m}$ each. The initial and boundary conditions can be framed by considering geometry of cell as follows.

Initial condition. Initially stable calcium concentration in hepatocyte cell is $0.1 \mu M$ [1]. Therefore, initial condition can be written as,

$$
C_{t=0}=0.1 \mu M, \quad \text { for } \quad 0 \leq x \leq 20, \quad 0 \leq y \leq 20, \quad t \geq 0 .
$$

Boundary conditions. As the calcium is released from channels located on membrane of endoplasmic reticulum, which are very dense near apical surface of a hepatocyte cell $[\underline{4}, \underline{25}]$. With this assumption, it is considered that calcium releasing channel is kept at $(0,10) \mu m$ situated near midway of apical surface. Therefore by using Fick's law of diffusion the first boundary condition can be set as;

$$
\lim _{x \rightarrow 0, y \rightarrow 10}-D_{C} \frac{\partial C}{\partial x}=\sigma_{C} \delta(x, y) \quad \text { for } \quad x=0, \quad 0 \leq y \leq 20, \quad t \geq 0,
$$

where, $\sigma_{C}$ is influx of calcium from calcium channel and $\delta(x, y)$ is Dirac delta function sharply peaked at source kept at point $(0,10)$.

On the remaining sides, other than the side having calcium channel of a hepatocyte cell, the calcium concentration attains its stable concentration $0.1 \mu M$. Therefore second boundary condition is framed along remaining sides of cells. It is assumed that, along remaining three sides calcium attains stable calcium concentration $\left(C_{\infty}\right)$ in a hepatocyte cell $[\underline{4}, \underline{26}]$.

$$
C=C_{\infty}=0.1 \mu M
$$

along the three boundaries, $x=20,0 \leq y \leq 20 \mu m, y=0,0 \leq x \leq 20 \mu m$, and $y=20$, $0 \leq x \leq 20 \mu m$. 


\section{SOLUTION}

The first step of finite volume method is discretization of domain under consideration i.e. hepatocyte cell. In two dimensions hepatocyte cell (represented by square) is discretised as shown in Fig. 2, by using uniform grid having 81 nodal points. The hepatocyte cell is divided into 9 control volumes along each of the $\mathrm{x}$ and $\mathrm{y}$ direction. The horizontal and vertical distances between cell faces are denoted by $\delta x$ and $\delta y$ [27] equal to $2.5 \mu \mathrm{m}$ each. The time step taken for numerical simulation is $\Delta t=1 \mathrm{msec}$.

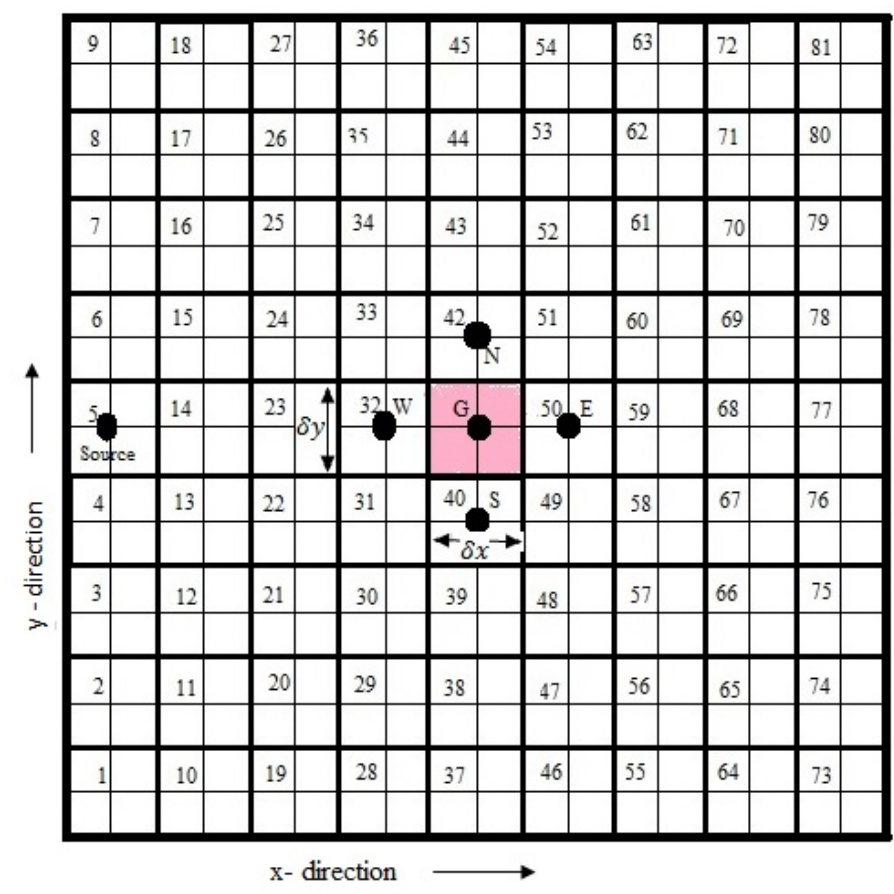

Fig. 2. Two dimensional discretization of hepatocyte cell.

Equation (1) can be rearranged as,

$$
\begin{gathered}
\frac{1}{D_{C}} \frac{\partial C}{\partial t}=\frac{\partial^{2} C}{\partial x^{2}}+\frac{\partial^{2} C}{\partial y^{2}}-\frac{u}{D_{C}} \frac{\partial C}{\partial x}-\frac{k_{j}^{+}\left[B_{j}\right]_{\infty}}{D_{C}}\left(C-C_{\infty}\right) \\
\text { for } 0 \leq x \leq 20,0 \leq y \leq 20, t \geq 0
\end{gathered}
$$

It can be written as,

$$
\begin{gathered}
\frac{1}{D_{C}} \frac{\partial C}{\partial t}=\frac{\partial^{2} C}{\partial x^{2}}+\frac{\partial^{2} C}{\partial y^{2}}-a \frac{\partial C}{\partial x}-b\left(C-C_{\infty}\right) \\
\text { for } 0 \leq x \leq 20,0 \leq y \leq 20, t \geq 0
\end{gathered}
$$

where, $a=\frac{u}{D_{C}}$ and $b=\frac{k_{j}^{+}\left[B_{j}\right]_{\infty}}{D_{C}}$

Now to apply second step of finite volume method, i.e discretization of equation over a control volume, integrating Eq.(6) w. r. to time $(t)$ and space $(x)$ gives, [23]

$$
\int_{t}^{t+\Delta t} \int_{x_{w}}^{x_{e}} \int_{y_{s}}^{y_{n}} \frac{1}{D_{C}} \frac{\partial C}{\partial t} d y d x d t=\int_{t}^{t+\Delta t} \int_{x_{w}}^{x_{e}} \int_{y_{s}}^{y_{n}}\left(\frac{\partial^{2} C}{\partial x^{2}}+\frac{\partial^{2} C}{\partial y^{2}}\right) d y d x d t
$$




$$
-a \int_{t}^{t+\Delta t} \int_{x_{w}}^{x_{e}} \int_{y_{s}}^{y_{n}} \frac{\partial C}{\partial x} d y d x d t-b \int_{t}^{t+\Delta t} \int_{x_{w}}^{x_{e}} \int_{y_{s}}^{y_{n}}\left(C-C_{\infty}\right) d y d x d t
$$

Solving space integration we get,

$$
\begin{gathered}
\frac{\delta x \delta y}{D_{C}} \int_{t}^{t+\Delta t} \frac{\partial C_{G}}{\partial t} d t=\int_{t}^{t+\Delta t}\left[\left(\frac{\partial C}{\partial x}\right)_{x_{e}}-\left(\frac{\partial C}{\partial x}\right)_{x_{w}}+\left(\frac{\partial C}{\partial y}\right)_{y_{n}}-\left(\frac{\partial C}{\partial y}\right)_{y_{s}}\right] d t \\
-a \delta y \int_{t}^{t+\Delta t}\left[C_{x_{e}}-C_{x_{w}}\right] d t-b \delta x \delta y \int_{t}^{t+\Delta t} C_{G} d t+b C_{\infty} \delta x \delta y \Delta t
\end{gathered}
$$

The weighted parameter $\theta$ is used to solve time integral.

$$
\begin{gathered}
\frac{\delta x \delta y}{D_{C}}\left[C_{G}-C_{G}^{0}\right]=\theta\left[\frac{C_{E}-C_{G}}{\delta x}-\frac{C_{G}-C_{W}}{\delta x}+\frac{C_{N}-C_{G}}{\delta y}-\frac{C_{G}-C_{S}}{\delta y}\right] \Delta t \\
+(1-\theta)\left[\frac{C_{E}^{0}-C_{G}^{0}}{\delta x}-\frac{C_{G}^{0}-C_{W}^{0}}{\delta x}+\frac{C_{N^{0}-C_{G}^{0}}}{\delta y}-\frac{C_{G}^{0}-C_{S}^{0}}{\delta y}\right] \Delta t \\
-a \delta y\left\{\theta\left[\frac{C_{G}+C_{E}}{2}-\frac{C_{W}+C_{G}}{2}\right]+(1-\theta)\left[\frac{C_{G}^{0}+C_{E}^{0}}{2}-\frac{C_{G}^{0}+C_{W}^{0}}{2}\right]\right\} \Delta t \\
\quad-b \delta x \delta y\left[\theta C_{G}-(1-\theta) C_{G}^{0}\right] \Delta t+b \delta x \delta y \Delta t
\end{gathered}
$$

where $\theta$ lies between 0 to 1 and the values of coefficient at old time $t$ are super scripted with 0 . The eq.(9) can be rearrange in the following form,

$$
\begin{gathered}
{\left[\frac{\delta x \delta y}{D_{C} \Delta t}+\frac{\theta}{\delta x}+\frac{\theta}{\delta x}+\frac{\theta}{\delta y}+\frac{\theta}{\delta y}+a \theta \delta x \delta y\right] C_{G}=} \\
{\left[\frac{\delta x \delta y}{D_{C} \Delta t}-\frac{(1-\theta)}{\delta x}-\frac{(1-\theta)}{\delta x}-\frac{(1-\theta)}{\delta y}+\frac{(1-\theta)}{\delta y}-a(1-\theta) \delta x \delta y\right] C_{G}^{0}} \\
+\left[\frac{\theta}{\delta x}-\frac{a \theta \delta y}{2}\right] C_{E}+\left[\frac{\theta}{\delta x}+\frac{a \theta \delta y}{2}\right] C_{W}+\left[\frac{\theta}{\delta y}\right] C_{N}+\left[\frac{\theta}{\delta y}\right] C_{S}+ \\
{\left[\frac{(1-\theta)}{\delta x}-\frac{a(1-\theta) \delta y}{2}\right] C_{E}^{0}+\left[\frac{(1-\theta)}{\delta x}+\frac{a(1-\theta) \delta y}{2}\right] C_{W}^{0}} \\
+\left[\frac{(1-\theta)}{\delta y}\right] C_{N}^{0}+\left[\frac{(1-\theta)}{\delta y}\right] C_{S}^{0}+b \delta x \delta y
\end{gathered}
$$

The Crank Nicolson scheme can be imposed by putting $\theta=1 / 2$ in Eq.(10),

$$
\begin{gathered}
{\left[\frac{\delta x \delta y}{D_{C} \Delta t}+\frac{1}{2}\left(\frac{1}{\delta x}+\frac{1}{\delta x}+\frac{1}{\delta y}+\frac{1}{\delta y}\right)+\frac{1}{2} a \delta x \delta y\right] C_{G}} \\
=\left[\frac{\delta x \delta y}{D_{C} \Delta t}-\frac{1}{2}\left(\frac{1}{\delta x}+\frac{1}{\delta x}+\frac{1}{\delta y}+\frac{1}{\delta y}\right)-\frac{1}{2} a \delta x \delta y\right] C_{G}^{0} \\
+\left[\frac{1}{2 \delta x}-\frac{a \delta y}{4}\right] C_{E}+\left[\frac{1}{2 \delta x}+\frac{a \delta y}{4}\right] C_{W}+\left[\frac{1}{2 \delta y}\right] C_{N}+\left[\frac{1}{2 \delta y}\right] C_{S}+ \\
{\left[\frac{1}{2 \delta x}-\frac{a \delta y}{4}\right] C_{E}^{0}+\left[\frac{1}{2 \delta x}+\frac{a \delta y}{4}\right] C_{W}^{0}+\left[\frac{1}{2 \delta y}\right] C_{N}^{0}+\left[\frac{1}{2 \delta y}\right] C_{S}^{0}+b \delta x \delta y}
\end{gathered}
$$

Eq.(11) can be put in the following form, for all internal nodes,

$$
\begin{gathered}
a_{G} C_{G}=a_{G}^{0} C_{G}^{0}+a_{E} C_{E}+a_{W} C_{W}+a_{N} C_{N}+a_{S} C_{S} \\
+a_{E} C_{E}^{0}+a_{W} C_{W}^{0}+a_{N} C_{N}^{0}+a_{S} C_{S}^{0}+S_{u}
\end{gathered}
$$

where,

$$
\begin{gathered}
a_{G}=\left[\frac{\delta x \delta y}{D_{C} \Delta t}+\frac{1}{2}\left(\frac{1}{\delta x}+\frac{1}{\delta x}+\frac{1}{\delta y}+\frac{1}{\delta y}\right)+\frac{1}{2} a \delta x \delta y\right] \\
a_{G}^{0}=\left[\frac{\delta x \delta y}{D_{C} \Delta t}-\frac{1}{2}\left(\frac{1}{\delta x}+\frac{1}{\delta x}+\frac{1}{\delta y}+\frac{1}{\delta y}\right)-\frac{1}{2} a \delta x \delta y\right] \\
a_{E}=\left[\frac{1}{2 \delta x}-\frac{a \delta y}{4}\right] ; a_{W}=\left[\frac{1}{2 \delta x}+\frac{a \delta y}{4}\right] \\
a_{N}=a_{S}=\frac{1}{2 \delta y} \\
S_{u}=b \delta x \delta y
\end{gathered}
$$


To incorporate first boundary condition at node 5, setting, $C_{W}=\sigma_{C}$

$$
a_{G} C_{G}=a_{G}^{0} C_{G}^{0}+a_{E} C_{E}+a_{N} C_{N}+a_{S} C_{S}+a_{E} C_{E}^{0}+a_{N} C_{N}^{0}+a_{S} C_{S}^{0}+S_{u}
$$

where,

$$
\begin{gathered}
a_{G}=\left[\frac{\delta x \delta y}{D_{C} \Delta t}+\frac{1}{2}\left(\frac{1}{\delta x}+\frac{1}{\delta y}+\frac{1}{\delta y}\right)+\left(\frac{1}{\delta x}+\frac{1}{2} a \delta x \delta y\right)\right] \\
a_{G}^{0}=\left[\frac{\delta x \delta y}{D_{C} \Delta t}-\frac{1}{2}\left(\frac{1}{\delta x}+\frac{1}{\delta y}+\frac{1}{\delta y}\right)-\left(\frac{1}{\delta x}+\frac{1}{2} a \delta x \delta y\right)\right] \\
a_{E}=\left[\frac{1}{2 \delta x}-\frac{a \delta y}{4}\right] ; a_{N}=a_{S}=\frac{1}{2 \delta y} \\
S_{u}=\left[\frac{2}{\delta x}+\frac{b}{2}\right] \sigma_{C}+b \delta x \delta y
\end{gathered}
$$

To incorporate second boundary condition at node 1 , setting $C_{\infty}=0.1$ and $a_{W}=a_{S}=0$ we get,

$$
a_{G} C_{G}=a_{G}^{0} C_{G}^{0}+a_{E} C_{E}+a_{N} C_{N}+a_{E} C_{E}^{0}+a_{N} C_{N}^{0}+S_{u}
$$

where,

$$
\begin{gathered}
a_{G}=\left[\frac{\delta x \delta y}{D_{C} \Delta t}+\frac{1}{2}\left(\frac{1}{\delta x}+\frac{1}{\delta y}\right)+\left(\frac{1}{\delta x}+\frac{1}{\delta y}+\frac{1}{2} a \delta x \delta y\right)\right] \\
a_{G}^{0}=\left[\frac{\delta x \delta y}{D_{C} \Delta t}-\frac{1}{2}\left(\frac{1}{\delta x}+\frac{1}{\delta y}\right)-\left(\frac{1}{\delta x}+\frac{1}{\delta y}+\frac{1}{2} a \delta x \delta y\right)\right] \\
a_{E}=\left[\frac{1}{2 \delta x}-\frac{a \delta y}{4}\right] ; a_{N}=\frac{1}{2 \delta y} \\
S_{u}=\left[\frac{2}{\delta x}+\frac{b}{2}\right] C_{B}+b \delta x \delta y
\end{gathered}
$$

Similarly, second boundary condition can be incorporate at all boundary nodes, 1 to 73,9 to 81 , and 73 to 81 . All equations obtained for each node forms the system of linear algebraic equations. Which can be expressed in matrix form as follows, for each time step;

$$
[A]_{81 \times 81}[C]_{81 \times 1}=[B]_{81 \times 1} .
$$

The Gauss elimination method is used to solve Eq.(15) at each time iteration to obtain solution vector $C_{81 \times 1}$ by developing a MATLAB program.

\section{RESULTS AND DISCUSSION}

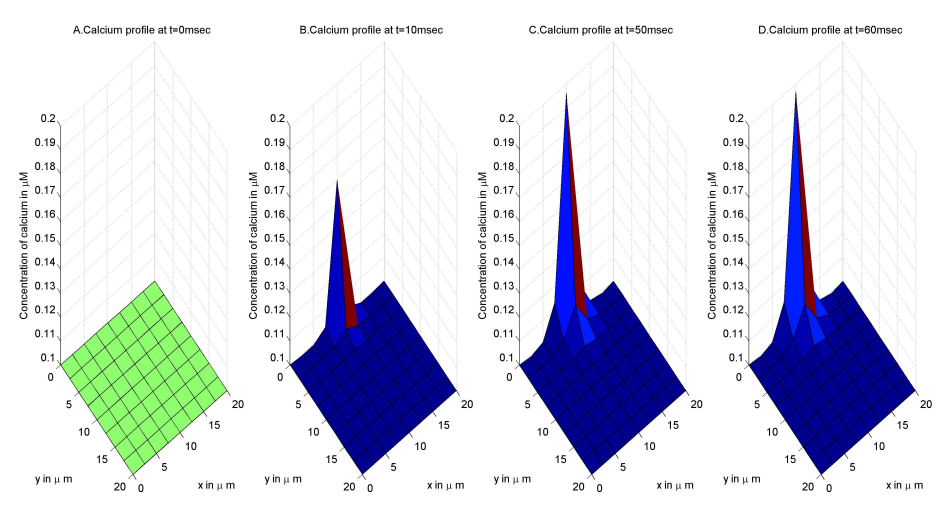

Fig. 3. The spatial variation of calcium profile in the presence of EGTA buffer.

The spatial variation of calcium in the presence of $50 \mu M$ EGTA buffer, with $10 \mu \mathrm{m} / \mathrm{sec}$ cross flow velocity is shown in Fig. 3 . The calcium concentration in hepatocyte cell at time $\mathrm{t}=0$, $10,50,60 \mathrm{~ms}$ is plotted in subplots A, B, C, D respectively. Initially before opening the mouth 
of calcium channel the calcium concentration is $0.1 \mu M$. After opening the mouth of calcium channel nodal calcium concentration increases with time. The nodal calcium concentration becomes constant after $t=50 \mathrm{~ms}$. Thus steady state concentration is achieved within $50 \mathrm{~ms}$ in the presence of $50 \mu \mathrm{M}$ EGTA buffer and $10 \mu \mathrm{m} / \mathrm{sec}$ cross flow velocity. The calcium concentration observed to be maximum at the mouth of calcium channel. It decreases sharply to attain stable concentration in remaining part of cell.

The effect of change in EGTA buffer concentration at constant cross flow velocity and diffusion coefficient is shown in Fig. 4. The calcium profile in a hepatocyte cell in the presence of $0,50,100 \mu M$ EGTA buffer concentration is plotted in subplots $\mathrm{A}, \mathrm{B}, \mathrm{C}$ respectively. The nodal calcium concentration is maximum in absence of any kind of buffer. It is higher for lower values of buffer concentration. The buffer binds with free calcium ions as soon as they are released from mouth of calcium channel. This results in decrease in nodal calcium concentration away from the mouth of calcium channel.
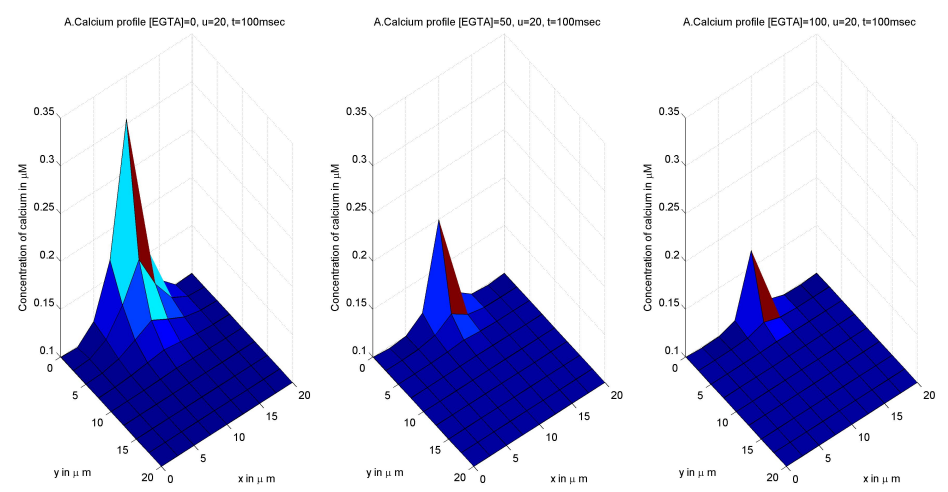

Fig. 4. Spatial variation of calcium profile at different EGTA buffer concentrations and constant cross flow velocity in the hepatocyte cell.
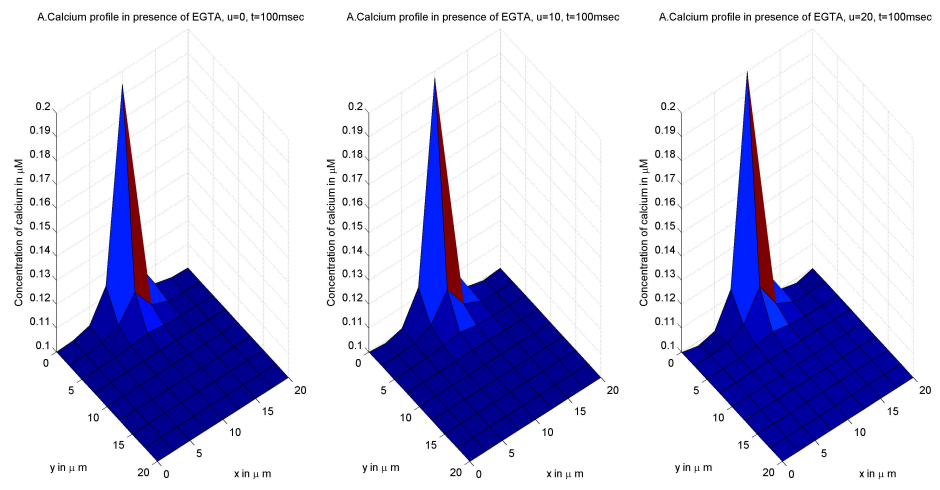

Fig. 5. The effect of cross flow velocity on spatial calcium profile in the presence of EGTA buffer in the hepatocyte cell.

The calcium concentration in hepatocyte cell with cross flow velocity $0,10,20 \mu \mathrm{m} / \mathrm{s}$ in the presence of $50 \mu M$ EGTA buffer is shown in subplots A, B and C of Fig. 5. respectively. We observed that the nodal concentration of calcium increases slightly with increase in cross flow velocity of calcium ions. 

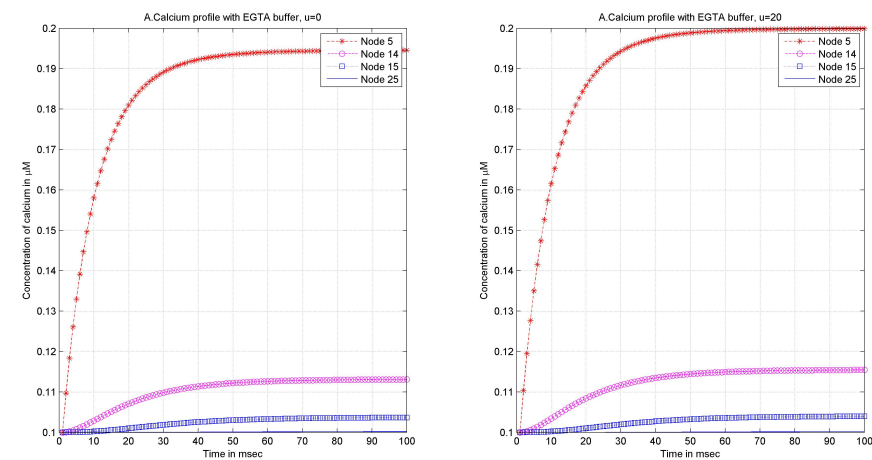

Fig. 6. The effect of cross flow velocity on temporal calcium profile in the presence of EGTA buffer.
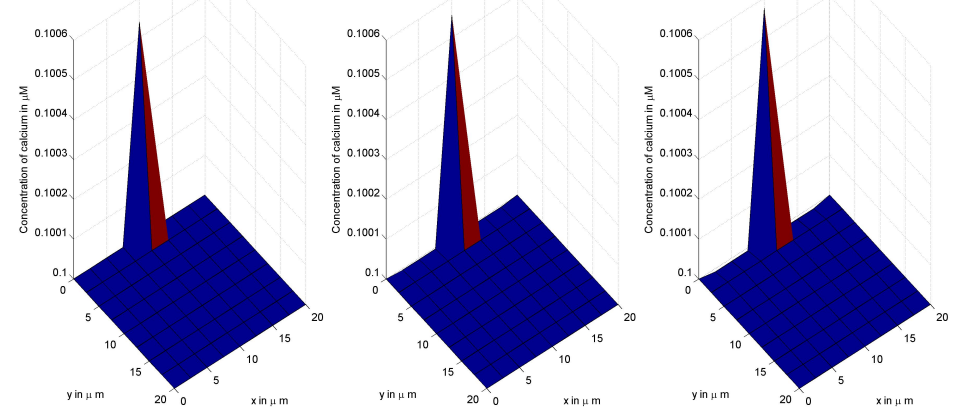

Fig. 7. The effect of cross flow velocity on calcium profile in the presence of BAPTA buffer.
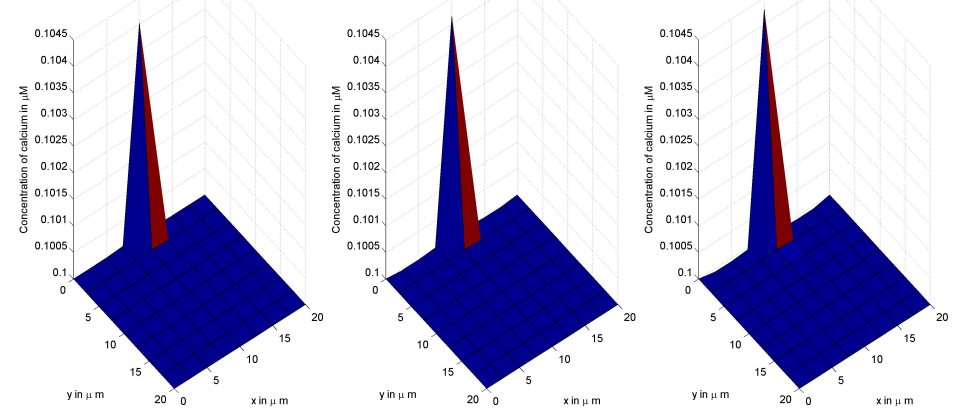

Fig. 8. The effect of cross flow velocity on calcium profile in the presence of endogenous buffer. 
The effect of cross flow velocity on nodal concentration is shown in Fig. 6. The graphs are plotted at node $5,14,15,25$. The increase in calcium concentration is sharp in vicinity of mouth of channel. It decreases sharply away from channel mouth. The effect of increased cross flow velocity is observed high in the region nearby the channel. It decreases with distance away from the mouth of channel.

The effect of cross flow velocity on calcium concentration in the presence of BAPTA buffer is shown in Fig. 7. The minimum nodal calcium concentration is seen in the presence of BAPTA buffer. This is due to high binding capacity of BAPTA buffer. As soon as calcium is released from mouth of calcium channel BAPTA buffer binds with calcium ions with in very short period. This leads to decrease in nodal calcium concentration. The increase in cross cross flow velocity has not shown any substantial change on calcium profile in the presence of BAPTA buffer.

The effect of cross flow velocity on calcium concentration in the presence of endogenous buffer is shown in Fig. 8. The calcium profile with cross flow velocity $0,10,20 \mu \mathrm{m} / \mathrm{s}$ is plotted in subplots A, B, C respectively. The calcium concentration increases slightly with increase in cross flow velocity in the presence of endogenous buffer.

The effect of change in value of diffusion coefficient of calcium in cytoplasm on calcium concentration is shown in Fig. 9. The calcium profile has been plotted by keeping constant cross flow velocity and constant EGTA buffer concentration with $D C=100,150,200 \mu \mathrm{m}^{2} / \mathrm{s}$. It is observed that as diffusion coefficient increases, the calcium concentration decreases simultaneously. This is because the increase in diffusion coefficient causes increase in transport of calcium ions from source channel to basal part of cell leading to decrease in calcium concentration in the cell.
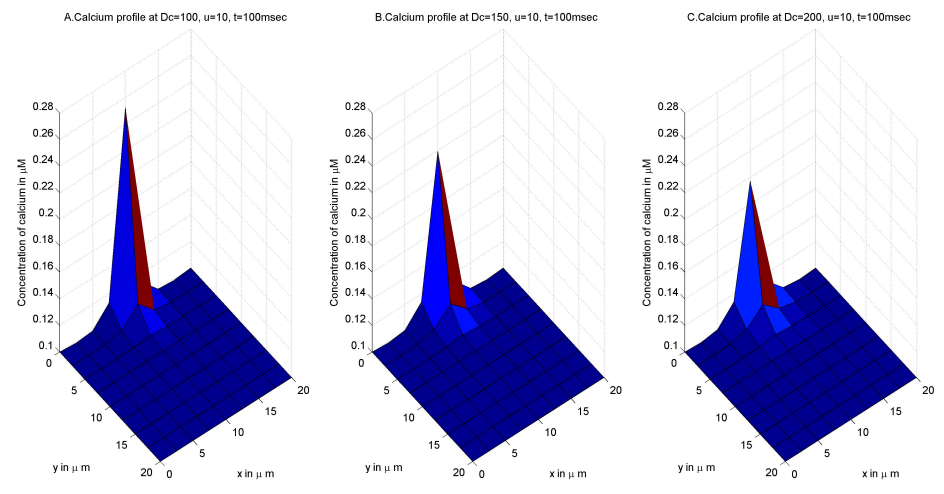

Fig. 9. The effect of diffusion coefficient on calcium profile in the presence of EGTA buffer.

\section{CONCLUSION}

The finite volume model is proposed and successfully implemented to study buffered advection diffusion in hepatocyte cell. The results obtained by simulation gives better insight of interrelationships among different physical transport mechanisms like, advection and diffusion in the presence of varieties of buffers. From the obtained results, it can be concluded that the advection mechanism greatly affects calcium concentration in the presence of EGTA buffer and endogenous buffer having small association rate constant, in the comparison with BAPTA buffer which has large association rate constant. The increase in cross flow velocity increases calcium concentration. While in contrast, increase in diffusion coefficient, decreases the calcium concentration in hepatocyte cell. Thus for high requirement of calcium concentration for specific activity, the advection mechanism is triggered by the cell to raise calcium concentration levels in the cell. Thereafter when activity is over the diffusion, buffering mechanism comes into play 
to reduce the calcium concentration in the cell to acceptable tolerable limits of cell. The finite volume method is proved to be a versatile tool in the present study. The proposed model is able to provide us the patterns of different combinations of transport mechanisms leading to specific spatial and temporal calcium patterns which are results of fine tuning and coordination of three transport mechanisms in response to requirements of the specific activity of the cell. Such models can be developed further to generate the spatial and temporal patterns of calcium concentration in the cell formed by fine coordination among transport mechanisms in response to specific activity. The information generated from such models will be of great use to understand the fine coordination of processes in the cell and disturbance in these physical coordination leading to diseases of liver.

\section{REFERENCES}

1. Rooney T.A., Sass E.J., Thomas A.P. Agonist-induced cytosolic calcium oscillations originate from a specific locus in single hepatocytes. Journal of Biological Chemistry. 1990. V. 265. P. 10792-10796.

2. Garcin I., Tordjmann T. Calcium signalling and liver regeneration. International Journal of Hepatology. 2012. V. 2012. P. 1-6.

3. Jafri M.S. Joel K. On the roles of $\mathrm{Ca}^{2+}$ diffusion, $\mathrm{Ca}^{2+}$ buffers, and the endoplasmic reticulum in IP3-induced $\mathrm{Ca}^{2+}$ waves. Biophysical Journal. 1995. V. 69. P. 2139-2153.

4. Dupont G. Swillens S. Clair C. Tordjmann T. Hierarchical organization of calcium signals in hepatocytes : from experiments to models. Biochimica et Biophysica Acta (BBA)-Molecular Cell Research. 2000. V. 1498. P. 134-152.

5. Sneyd J. Calcium buffering and diffusion: on the resolution of an outstanding problem. Biophysical Journal. 1994. V. 67. P. 4.

6. Tewari S. Pardasani K.R. Finite element model to study two dimensional unsteady state cytosolic calcium diffusion in presence of excess buffers. IAENG International Journal of Applied Mathematics. 2010. V. 40. P. 108-112.

7. Jha A. Adlakha N. Finite element model to study the effect of exogenous buffer on calcium dynamics in dendritic spines. International Journal of Modeling, Simulation, and Scientific Computing. 2014. V. 5. P.1350027.

8. Kotwani M. Adlakha N. Modeling of endoplasmic reticulum and plasma membrane $\mathrm{Ca}^{2+}$ uptake and release fluxes with excess buffer approximation (EBA) in fibroblast cell. International Journal of Computational Materials Science and Engineering. 2017. V.6 .P. 1750004.

9. Kotwani M. Adlakha N. Finite element model to study the effect of buffers, source amplitude and source geometry on spatio-temporal calcium distribution in fibroblast cell. Journal of Medical Imaging and Health Informatics. 2014. V. 4. P. 840-847.

10. Jha B.K. Adlakha N. Mehta M.N. Two-dimensional finite element model to study calcium distribution in astrocytes in presence of VGCC and excess buffer. Int. J. Model. Simul. Sci. Comput. 2013. V. 4. P. 1250030.

11. Jha B.K. Adlakha N. Mehta M.N. Two-dimensional finite element model to study calcium distribution in astrocytes in presence of excess buffer. International Journal of Biomathematics. 2014. V. 7. P. 1450031.

12. Naik P.A. Pardasani K.R. One Dimensional Finite Element Model to Study Calcium Distribution in Oocytes in Presence of VGCC, RyR and Buffers. J. Medical Imaging Health Informatics. 2015. V. 5. P. 471-476.

13. Pathak K. Adlakha N. Finite Element Model to Study Calcium Signaling in Cardiac Myocytes Involving Pump, Leak and Excess Buffer. Journal of Medical Imaging and Health Informatics. 2015. V. 5. P. 1-6. 
14. Pathak K. Adlakha N. Finite element model to study two dimensional unsteady state calcium distribution in cardiac myocytes. Alexandria Journal of Medicine. 2016. V. 52. P. 261-268.

15. Jagtap Y.D. Adlakha N. Finite volume simulation of two dimensional calcium dynamics in a hepatocyte cell involving buffers and fluxes. Commun. Math. Biol. Neurosci. 2018. V.2018. P. 1-16.

16. Jha A. Adlakha N. Two-dimensional finite element model to study unsteady state $\mathrm{Ca}^{2+}$ diffusion in neuron involving ER LEAK and SERCA. International Journal of Biomathematics. 2015. V. 89. P. 1550002.

17. Naik P.A. Pardasani K.R. One dimensional finite element method approach to study effect of ryanodine receptor and serca pump on calcium distribution in oocytes. Journal of Multiscale Modelling. 2013. V. 5. P. 1350007.

18. Panday S. Pardasani K.R. Finite element model to study the mechanics of calcium regulation in oocyte. Journal of Mechanics in Medicine and Biology. 2014. V. 14. P. 1450022.

19. Manhas N. Pardasani K.R. Modelling mechanism of calcium oscillations in pancreatic acinar cells. Journal of Bioenergetics and Biomembranes. 2014. V. 46. P. 403-420.

20. Manhas N. Sneyd J. Pardasani K.R. Modelling the transition from simple to complex $\mathrm{Ca}^{2+}$ oscillations in pancreatic acinar cells. Journal of Biosciences. 2014. V. 39. P. 463-484.

21. Jha B. Adlakha N. Mehta M.N. Finite volume model to study the effect of buffer on cytosolic $\mathrm{Ca}^{2+}$ advection diffusion. Int. J. of Eng. and Nat. Sci. 2010. V. 4. P.60-163.

22. Pathak K. Adlakha N. Finite Element Simulation of Advection Diffusion of Calcium in Myocyes Involving Influx and Excess Buffer. Advances in Computational Sciences and Technology. 2017. V. 10. P. 11-23.

23. Panday S. Pardasani K.R. Finite Element Model to Study Effect of Advection Diffusion and $\mathrm{Na}^{+} / \mathrm{Ca}^{2+}$ Exchanger on $\mathrm{Ca}^{2+}$ Distribution in Oocytes. Journal of Medical Imaging and Health Informatics. 2013. V. 3. P. 374-379.

24. Keener J.P. Sneyd J. In: Mathematical physiology. Springer, 1998. P. 309-313.

25. In: Calcium: The molecular basis of calcium action in biology and medicine. Eds. R. Pochet, R. Donato, J. Haiech, C.W. Heizmann, V. Gerke. Springer Science \& Business Media, 2011. V. 3. P. 73-94.

26. Thomas A.P. Bird GSTJ. Hajnoczky G. Gaspers R. Spatial and temporal aspects of cellular calcium signaling. The FASEB Journal. 1996. P. 1505-1517.

27. Versteeg H.K., Malalasekera W. In: An introduction to computational fluid dynamics: the finite volume method. Pearson Education, 2007. 